Int. J. Odontostomat.,

6(2):181-187, 2012.

\title{
Salud Bucodental de Postrados Severos
}

\author{
Oral Health Status of Elderly Bedridden
}

Daniela Muñoz A.; Pricila Flores A."*; Alfredo Cueto U..* \& Alan Barraza S. ${ }^{* \star * *}$

MUÑOZ, A. D.; FLORES, A. P.; CUETO, U. A. \& BARRAZA, S. A. Salud bucodental de postrados severos. Int. J. Odontostomat., 6(2):181-187, 2012.

RESUMEN: El propósito de esta investigación fue describir la salud oral en base a COP, PSR, desdentamiento, lesiones de mucosa, uso de prótesis e higiene bucal en pacientes postrados. Se realizó un estudio transversal con una muestra probabilística con afijación proporcional, según distribución geográfica y sexo, obtenida del listado de los inscritos en el Programa deAtención Domiciliaria a Postrados de la Corporación Municipal de Valparaíso. A los pacientes se les sometió a una entrevista y un examen bucodentario. Se examinaron 78 pacientes postrados severos, $64,1 \%$ mujeres y $35,9 \%$ hombres de una edad promedio 83,9 y $79,7$ años respectivamente. El COP registrado fue 29,91( $\pm 2,52)$ (IC del 95\%= [29,06 - 30,76]); con un índice de cariados 1,60; obturados 0,51 ; y perdidos, 27,79 . El estado periodontal encontrado fue altamente deteriorado con $78,8 \%$ de dientes con pérdida de inserción clínica mayor a 3,5 mm, 15,2\% de código 4; 36,5\% de código 3; y 48,5\% de código 2 de PSR. Las lesiones de tejidos blandos afectaron al $52,5 \%$ de la muestra, siendo más prevalente el tipo vascular (lago venoso) con un $25 \%$, seguida por $23,21 \%$ de estomatitis subprotésica. El 51,3\% fue desdentado total. En cuanto a distribución dentaria, en maxilar y mandíbula, el hallazgo más frecuente fue Clase I de Kennedy (50\% maxilar y $63,9 \%$ mandibular). Sólo el $43,6 \%$ usaba sus prótesis, la mayoría totales acrílicas (77,8\% maxilar y $54,5 \%$ mandibular). Se encontró un estado extremadamente deteriorado de salud bucal en este grupo, con escasas posibilidades de ser recuperado, principalmente debido a las disminuidas condiciones sistémicas que presentan y a la falta de cobertura de los programas de salud bucal para estas personas.

PALABRAS CLAVE: postrados, diagnóstico, oral, bucal.

\section{INTRODUCCIÓN}

Chile experimenta un envejecimiento demográfico avanzado; actualmente el $13 \%$ de la población está constituida por adultos mayores, y de estos un $12,4 \%$ presenta dependencia severa (SENAMA - INTA, 2009). Los estudios realizados a nivel país se refieren escasamente a la salud bucodental de los pacientes adultos mayores postrados y por otra parte, la atención de salud que se le brinda al paciente inmovilizado en el marco del Programa Postrados del MINSAL (2003) no incluye diagnóstico ni tratamiento de las patologías bucodentales, aun cuando este factor de riesgo es conocido como importante para la salud sistémica (Petersen \& Yamamoto, 2005). De ahí la importancia de realizar un estudio de las condiciones bucales de los postrados en la región, y evidenciar las particulares necesidades de atención odontológica de estos pacientes.
Las características de salud general de los adultos mayores, propias del ciclo vital están dadas por un deterioro de la funcionalidad, por una pérdida de respuesta adaptativa al estrés asociada a la edad (Kirkwood \& Ritter, 1996), lo que en muchos casos da inicio a un ciclo de discapacidad el cual parte por la deficiencia, sigue con limitaciones funcionales y restricción en la participación culminando en dependencia en diferentes niveles, y ésta se describe como la necesidad de ayuda de otros para realizar las actividades de la vida diaria de una persona (OMS, 2001).

El nivel más grave es la postración o dependencia severa, significa una salud general francamente disminuida asociada comúnmente a polifarmacia y dificultad en el traslado del postrado hacia centros asistenciales lo que perpetúa condiciones patológicas

* Cirujano dentista. Profesora ayudante de la Facultad de Odontología, Universidad de Valparaíso, Valparaíso, Chile.

** Interna de la Facultad de Odontología, Universidad de Valparaíso, Valparaíso, Chile.

**** Cirujano dentista. Profesor titular de la Facultad de Odontología, Universidad de Valparaíso, Valparaíso, Chile.

${ }^{* * * *}$ Estadístico. Profesor ayudante de la Facultad de Odontología, Universidad de Valparaíso, Valparaíso, Chile. 
y de riesgo, como lesiones de mucosa bucal, ya sea como manifestaciones orales de condiciones sistémicas o producto de condiciones locales tales como: infección, procesos tumorales, lesiones por mal ajuste protésico, alteración de las funciones del sistema estomatognático entre otras, que resultan en una deteriorada salud bucal que a su vez puede agravar aún más las condiciones sistémicas ya disminuidas. Dicho estado además trae repercusiones considerables para el núcleo familiar tanto en el ámbito social como el económico.

Al crecer la población de adultos mayores, y por ende los postrados, creemos relevante conocer cómo se encuentra la salud de esta población, pues la información disponible es escasa a nivel nacional e inexistente a nivel regional, convirtiéndose este estudio en pionero en enfrentar esta temática a nivel comunal, lo que podría orientar a la autoridad en salud para el desarrollo eventual de programas de salud bucodental que beneficien a este grupo vulnerable de la sociedad. El objetivo de esta investigación fue describir la salud oral de los adultos mayores postrados severos de la comuna de Valparaíso.

\section{MATERIAL Y MÉTODO}

Estudio de prevalencia. La población en estudio fueron los inscritos en el Programa de Atención Domiciliaria a Postrados de la Corporación Municipal de Valparaíso. Sobre esta población, se calculó la muestra considerando un $5 \%$ de error de estimación, para ajustarla se utilizó la prevalencia de un $29,1 \%$ de desdentados totales nacional para el rango mayor de 65 años obtenido de la Encuesta Nacional de Salud 2003. Se utilizó muestreo estratificado con afijación proporcional seleccionándose aleatoriamente cada participante de acuerdo a género y centro de salud con inscritos en el Programa, obteniendo una muestra de 78 personas.

La recolección de datos se realizó mediante visitas domiciliarias que consideraba una entrevista al paciente, a su cuidador y un examen bucodental al paciente postrado con registro de periodontograma completo.

Se midieron variables epidemiológicas: género, edad, nivel educacional; variables de salud general: enfermedades sistémicas concomitantes y consumo de fármacos; $y$ variables bucodental: COP con base de 32 dientes, índice PSR, desdentamiento, lesiones de mucosa bucal, topografía dentaria según clases de Kennedy (Applegate, 1960), frecuencia de aseo bucal, índice de higiene oral de O'Leary, frecuencia de cepillado dental. Además, en los pacientes desdentados, aseo de las mucosas con gasa o toallas húmedas y aseo de prótesis. Los exámenes fueron realizados por un solo examinador entrenado y calibrado clínicamente (Kappa=0,8 tanto en caries como en enfermedad periodontal) y en el caso de las lesiones bucales, la calibración se realizó por medio de revisión de fotografías clínicas hasta obtener consistencia aceptable. Se calculó el índice de necesidades de tratamiento para caries el que se obtiene al dividir la cantidad de dientes que requieren tratamiento por caries por el total de dientes en boca. Se obtuvo consentimiento informado de los participantes en cada caso que estaban en condición cognitiva de otorgarlo o en su defecto de los cuidadores (tutores legales). El protocolo del estudio fue previamente aprobado por el Comité de Ética de la Facultad de Odontología.

Se construyó una base de datos en Excel 2010 y se realizó el análisis mediante estadísticas descriptivas. Para corroborar si existía asociación entre variables se utilizó el test exacto de Fisher y la correlación de Pearson en el programa Minitab 15.

\section{RESULTADOS}

Se estudiaron 78 personas postradas severas, de los cuales $50(64,1 \%)$ fueron mujeres y $28(35,9 \%)$ hombres, todas de un nivel socioeconómico bajo y

Tabla I. Características epidemiológicas y de salud general.

\begin{tabular}{lcccccc}
\hline & Promedio & DE & Mediana & Moda & Mínimo & Máximo \\
\hline Edad & 82,40 & 8,38 & 84 & 86 & 65 & 97 \\
Años de educación & 7,19 & 4,06 & 6 & 6 & 0 & 17 \\
Meses de postración & 70 & 106 & 36 & 36 & 0 & 720 \\
Enfermedades sistémicas & 2,97 & 1,65 & 3 & 3 & 0 & 8 \\
Consumo diario de fármacos & 3,51 & 2,24 & 3,5 & 4 & 0 & 11 \\
\hline
\end{tabular}


MUÑOZ, A. D.; FLORES, A. P.; CUETO, U. A. \& BARRAZA, S. A. Salud bucodental de postrados severos. Int. J. Odontostomat., 6(2):181-187, 2012.

Tabla II. Estadística descriptiva de dientes cariados, obturados y perdidos en maxilar y mandíbula.

\begin{tabular}{lccccccc}
\hline & Promedio & IC 95\% & DE & Mediana & Moda & Mínimo & Máximo \\
\hline Cariados & 1,60 & $1,03-2,17$ & 2,52 & 0 & 0 & 0 & 10 \\
Obturados & 0,51 & $0,23-0,79$ & 1,24 & 0 & 0 & 0 & 6 \\
Perdidos & 27,79 & $26,43-29,16$ & 6,04 & 31 & 32 & 11 & 32 \\
COP & 29,91 & $29,06-30,76$ & 3,77 & 32 & 32 & 14 & 32 \\
\hline
\end{tabular}

pertenecientes a FONASA A o B. Las características epidemiológicas y de salud general se observan en la Tabla I.

Tabla III. Prevalencia de lesiones de tejidos blandos.

\begin{tabular}{lcc}
\hline & $\mathbf{n}$ & $\%$ \\
\hline Lago venoso & 14 & 25,00 \\
Estomatitis & 13 & 23,21 \\
Queilitis angular & 12 & 21,43 \\
Úlceras & 5 & 8,93 \\
Fibroma & 3 & 5,36 \\
Melanosis & 2 & 3,57 \\
Candidiasis pseudomembranosa & 1 & 1,79 \\
Épulis fisurado & 1 & 1,79 \\
Herpes labial & 1 & 1,79 \\
Hiperqueratosis lengua & 1 & 1,79 \\
Lengua pilosa (glosofitia) & 1 & 1,79 \\
Leucoplaquia en lengua & 1 & 1,79 \\
Queilitis actínica & 1 & 1,79 \\
Total & 56 & 100,00 \\
\hline
\end{tabular}

La distribución de dientes cariados, obturados y perdidos, se observa en la Tabla II. El índice de necesidades de tratamiento para caries fue de $38,67 \%$, para maxila alcanzó un $48,04 \%$ y mandíbula un $34,50 \%$. El estado periodontal arrojó un $48,5 \%$ de bocas codificadas con PSR 2, 36,3\% con PSR 3 y $15,4 \%$ con PSR 4. Un $78,8 \%$ de los individuos tuvo uno o más dientes con pérdida de inserción mayor a $3,5 \mathrm{~mm}$.

Las lesiones de tejidos blandos afectaron al $52,6 \%$ de la muestra (Tabla III).

Se encontró $51,3 \%$ de desdentados totales y $48,7 \%$ de desdentados parciales, el número promedio de dientes por persona fue de $3,6( \pm 5,6)$ (IC del $95 \%=[2,3-4,8])$. La distribución de dientes remanentes según Clasificación de Kennedy se observa en la Figura 1.

El uso de prótesis removible fue de $43,6 \%$, que corresponden a 34 pacientes que portaban 60 prótesis que se distribuían en: totales acrílicas (42 prótesis), parciales metálicas (9) y parciales acrílicas (9). Y del total de pacientes, 11 tenían juego de prótesis to-

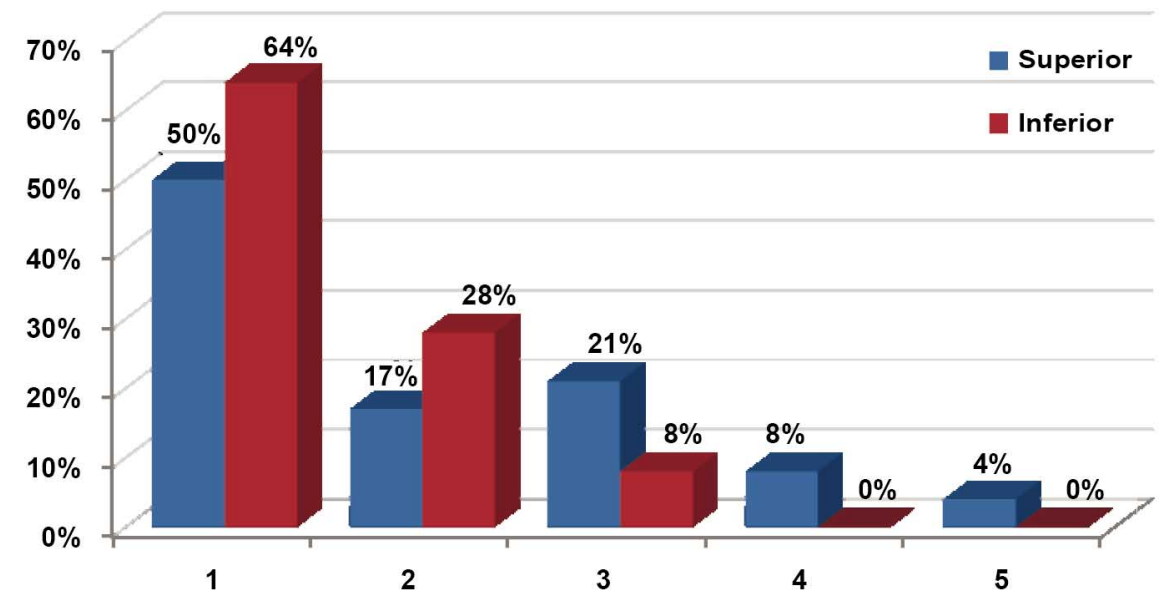

Fig. 1. Distribución de dientes remanentes según la Clasificación de Kennedy en maxilar y mandíbula. 
MUÑOZ, A. D.; FLORES, A. P.; CUETO, U. A. \& BARRAZA, S. A. Salud bucodental de postrados severos. Int. J. Odontostomat., 6(2):181-187, 2012.

Tabla IV. Tiempo de uso prótesis en años

\begin{tabular}{lccccccc}
\hline & Promedio & DE & Mínimo & Máximo & $\mathbf{0 - 1 0}$ años & $\mathbf{1 1 - 2 0}$ años & $\mathbf{2 0 - 4 2}$ años \\
\hline Maxilar & 6,79 & 10,6 & 0 & 42 & $52,7 \%$ & $30,6 \%$ & $16,7 \%$ \\
Mandibular & 3,42 & 6,82 & 0 & 30 & $54,6 \%$ & $36,4 \%$ & $9,0 \%$ \\
\hline
\end{tabular}

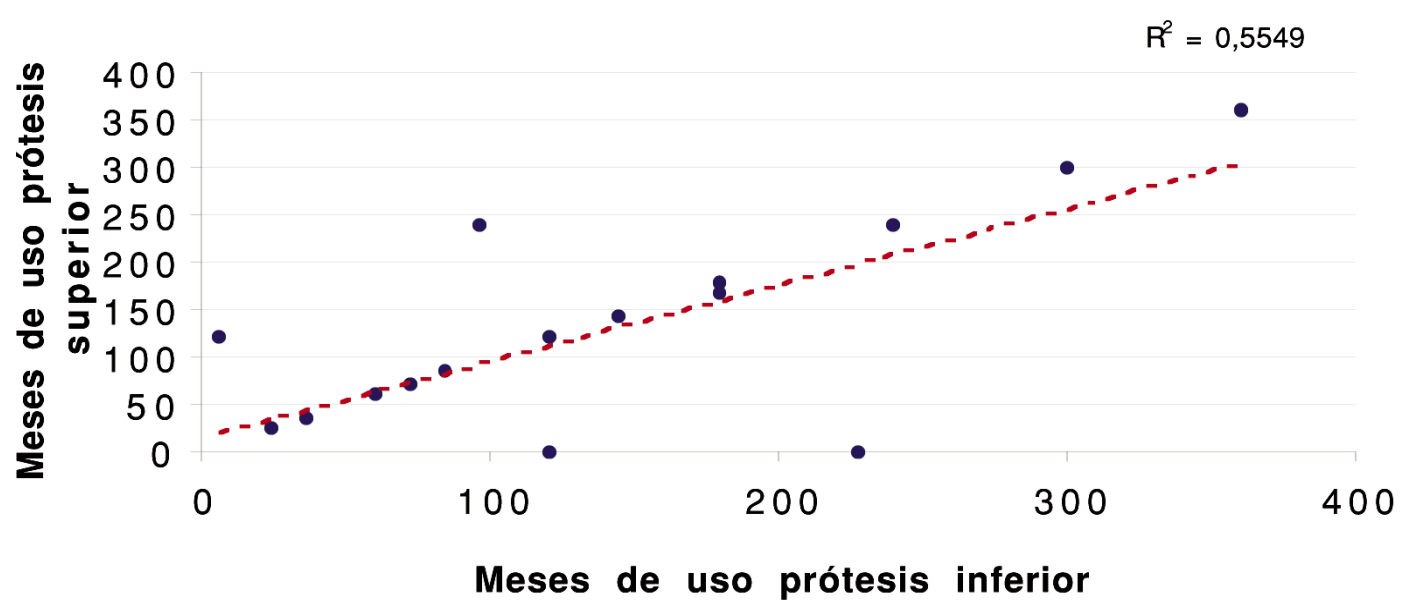

Fig. 2. Dispersión del tiempo de uso de protesis maxilar y mandibular.

tales. La descripción del tiempo de uso de protésis en años se observa en la Tabla IV, y su dispersión en término de tiempo se grafica en la Figura 2.

El tiempo de postración con el tiempo de uso de prótesis superior no presentó relación lineal (rho $=0,054 ; p$-valor $=0,75$ ), a su vez, el tiempo de postración con el tiempo de uso de prótesis inferior tampoco presentó relación lineal $(r h o=-0,329 ; p$-valor= $0,101)$.

Las prácticas de higiene oral en adultos mayores postrados son realizadas por sus cuidadores, un 29,5\% declaró realizarla sólo una vez al día, un 29,5\% que la realizaba 2 o más veces al día, mientras que el $41,1 \%(n=32)$ no se le realizaba limpieza bucal. La frecuencia de higiene de la prótesis en estos pacientes se distribuye entre: nunca $(25,6 \%)$, una vez al día $(25,6 \%)$, dos veces al día $(20,9 \%)$ y tres veces al día $(23,3 \%)$. El índice de higiene oral de O'Leary alcanzó un promedio de $100 \%$ en esta población.

\section{DISCUSIÓN}

Sin duda, uno de los hallazgos principales de la investigación en terreno fue la condición de salud bucal extremadamente deteriorada de nuestro grupo de estudio. Si bien es cierto anticipábamos este deterioro pues la literatura hacía referencia al daño acumulado en la boca en éstos pacientes (Meurman \& Hämäläinen, 2006), la magnitud del dicho daño en este estudio resultó mayor, pues en esta comuna los postrados se encuentran en un estado de abandono extremo en cuanto a salud oral, sin acceso a la Odontología, representando un desafío para los programas de salud.

El valor del índice COP promedio indica que la actividad cariogénica histórica, en estos pacientes es muy alta tanto al compararla con un estudio realizado en la región de Valparaíso, que examinó a 354 adultos mayores (Mariño et al., 2007), donde el COP alcanzo un valor de 17,5 , como en estudios de postrados desarrollado en la ciudad de Talca que obtienen valores de COP de 25,7 (Núñez \& Aspillaga, 2006; Núñez \& Quinteros, 2007), lo que demuestra que esta población está en total abandono odontológico.

Las pérdidas dentarias hacen el mayor peso en el COP en esta muestra, de hecho representan el $92,9 \%$ del total del índice, lo que se corrobora con el alto nivel de desdentamiento total tanto superior como 
inferior que alcanza el $51,3 \%$, superando ampliamente lo reportado por Mariño et al., que alcanzó un 30,5\%; esta condición de dientes perdidos numerosos es un factor de riesgo para problemas nutricionales en las personas mayores lo cual podría contribuir a complicar aún más su salud general.

Los dientes presentes en boca promedio eran 3,6 , de estos cerca de la mitad 1,6 estaban cariados, esta es una situación alarmante, ya que son focos infecciosos activos, actuando como nichos ecológicos bacterianos, lo cual implica un riesgo latente de gran magnitud, de bacteremia y constituyen un factor de riesgo para múltiples enfermedades relacionadas con un mal estado de salud bucal (Wilson et al., 2008; Azarpazhooh \& Leake, 2006; Awano et al., 2008). El bajo porcentaje de dientes obturados se puede interpretar como una escasa asistencia al odontólogo, y por otro lado puede representar un fracaso de la odontología restauradora. En definitiva este grupo se mantiene con un alto riesgo de caries pues su índice de O'Leary es de un $100 \%$, es decir, todos los pacientes con franca mala higiene oral, ellos al estar incapacitados y sus cuidadores al no efectuar una higiene adecuada los mantiene en permanente riesgo haciendo un pésimo pronostico su salud oral.

Desde el punto de vista periodontal los dientes remanentes presentan periodontos reducidos reflejo de daño acumulado. En el estudio de Mariño et al., que examinó el periodonto de 246 adultos mayores, encontró presencia de sacos en el $43,1 \%$ del total de personas, en cambio en nuestro estudio se constató mayor enfermedad periodontal $(57,1 \%)$. Según nuestra búsqueda bibliográfica no encontramos estudios con índices de enfermedad periodontal en población postrada en Chile lo que impide la comparación, y destaca la relevancia de este antecedente. Por otra parte, en esta muestra 14 pacientes seleccionados al azar que debían ser examinados no fue posible por estar hospitalizados por neumonía, enfermedad de alta prevalencia en postrados (Superintendencia de Salud, 2006). La condición periodontal precaria constituye un factor de riego para esta enfermedad sistémica lo cual esta ampliamente respaldado en la literatura (Awano et al.; Azarpazhooh \& Leake) y en consecuencia el daño periodontal podría estar subestimado en nuestra muestra.

Espinoza et al. (2003) examinó la mucosa oral de 889 adultos mayores sin dependencia que pertenecían a los sistemas salud público y privado de Santiago, hallando un $22,3 \%$ de estomatitis subprotésica coincidente con el porcentaje encontrado en nuestro estudio; esperábamos que la frecuencia de estomatitis fuera mayor en nuestro grupo sin embargo hay un número significativo de pacientes que no usa sus prótesis o que no tiene prótesis. Otras lesiones significativas detectadas fueron queilitis angular, úlceras, fibromas, incluso melanosis y leucoplaquia, éstas y las primeras no habían sido detectadas ni por el postrado que estaba en adecuadas condiciones cognitivas, ni por su cuidador, ni por el personal que otorga la asistencia sanitaria, si bien es cierto son de difícil diagnóstico para la población, algunas de estas lesiones se pueden considerar precancerígenas (Mariño et al.) y en consecuencia debería al menos incorporarse en forma urgente el examen de la mucosa oral en la visita habitual del personal de salud que cubre el programa de atención domiciliaria a postrados.

Se evalúo el tiempo de uso de la última prótesis que estaba aparentemente relacionado con el tiempo de postración. Los postrados caracterizados en esta muestra por muy bajo nivel socioeconómico lo cual les genera una barrera adicional de acceso a la atención odontológica domiciliaria, en su defecto la única atención dental que accedían era la otorgada por laboratoristas dentales que concurrían a los domicilios a confeccionar prótesis acrílicas, quienes no reparaban en las condiciones bucales previa a la instalación de la prótesis, encontrándose en la práctica retenedores dentro de caries y cuidadores no entrenados en indicaciones post instalación y éstos por no manipular la prótesis y evitar dañar al postrado agudizaban las malas condiciones de higiene oral.

Es necesario entrenar a los cuidadores de los pacientes postrados en técnicas de higiene bucal, pues un número significativo de pacientes a su cargo no se les realiza ningún tipo de higiene oral $(41,1 \%)$. Estos cuidados deberían respaldarse en un protocolo de higiene accesible, especialmente en pacientes con deficiencias neurológicas de difícil manejo, como aquellos que han desarrollado el reflejo de succión, lo cual impide un aseo correcto por parte de los cuidadores no entrenados e incluso dificulta la visualización de posibles lesiones que estén afectando la mucosa bucal de estos pacientes, incidiendo consecuentemente en un mayor deterioro de la salud bucal de los postrados (Shimovama et al., 2007).

La recogida de datos se basó en las respuestas proporcionadas por el cuidador, en ocasiones estas respuestas no reflejan la realidad y en este caso 
se sobrestiman los cuidados en salud oral, constituyéndose esta en una limitación por la técnica utilizada. Un estudio realizado en cuidadores de adultos mayores institucionalizados en Japón (Shimovama et al.), revela que si bien la mayoría de los cuidadores reconoce que los adultos mayores dependientes necesitan de cuidados especiales de su salud oral, e incluso un $77 \%$ reconoció tener entrenamiento en este tema, solo un $44 \%$ se sentía capacitado para proveer dicho cuidado de forma adecuada. El mismo estudio reveló diferencias significativas en la eficiencia del cuidado en aquellos con y sin entrenamiento en salud bucal, pero por otra parte, reconoció que la enseñanza teórica de los métodos de higiene en estos pacientes no fue suficiente, y es necesario contar con el apoyo de profesionales de la salud bucal.

En la higiene también hay que considerar el tiempo de uso de los aparatos protésicos; ha sido demostrado en varias poblaciones que los adultos mayores tienden a creer en la longevidad de sus tratamientos de prótesis removibles y a no recambiar sus aparatologías por períodos que superan los diez años (Ribeiro et al., 2009; de Castellucci et al., 2008). Este largo uso trae aparejado un deterioro del material de las prótesis, evidenciándose desgastes y porosidades donde se facilita la acumulación de restos alimenticios, tinciones y biofilm constituyendo un factor de riesgo de infección y lesiones.

Existe un gran deterioro de la salud oral de los postrados, caracterizado por: índice COP especialmente alto influenciado por un alto nivel de pérdidas dentarias, por el reducido estado periodontal, por el alto nivel de lesiones de mucosa oral, por las prácti- cas deficientes de higiene bucal y falta de acceso a tratamientos en especial rehabilitación con prótesis, esto en parte se explica porque los postrados de este estudio eran en general individuos muy longevos, con nivel socioeconómico bajo, con estados sistémicos deteriorados y con falta de acceso a la salud.

En definitiva los pacientes postrados están incapacitados de su aparato estomatognático lo que representa un fracaso de las políticas de salud bucal, que se han basado en una odontogeriatría mutiladora.

En conclusión, se encontró un estado de salud bucal extremadamente deteriorado en los postrados, con escasas posibilidades de ser recuperado, principalmente debido a las disminuidas condiciones del terreno biológico bucal sumado a condiciones sistémicas concomitantes graves y a la falta de cobertura en salud bucal en los programas de salud para adultos mayores.

Los investigadores proponen que se incorpore la Odontología en forma urgente al programa de atención domiciliaria de postrados del MINSAL con un enfoque preventivo, que además incluya tratamientos básicos de desfocación y rehabilitación protésica para así mejorar la salud oral de estas personas lo que contribuirá notablemente a su calidad de vida.

Se sugieren nuevas investigaciones sobre esta población, aplicando otros diseños de estudios como de seguimiento o de intervenciones para evaluar diferentes medidas terapéuticas y su efecto en su calidad de vida.

MUÑOZ, A. D.; FLORES, A. P.; CUETO, U.A. \& BARRAZA, S. A. Oral health status of elderly bedridden.Int. J. Odontostomat., 6(2):181-187, 2012.

ABSTRACT: The purpose of this study was to describe the oral health in bedridden patients on the basis of COP, PSR, toothlessness, mucosal injuries, prothesis and mouth hygiene. A transverse study with a probabilistic sample with proportional fixation, according to geographical distribution and gender, obtained of the list of the people in the Program of Domiciliary Attention to Bedridden of the Municipal Corporation of Valparaiso. The patients were interviewed and examinated. We examined 78 severely bedridden patients, $64.1 \%$ women and $35.9 \%$ men of an average age 83.9 and 79.7 years respectively. The registered COP was $29.91( \pm 2.52)($ IC of $95 \%=$ [29.06 - 30.76]); with an index of decay 1.60; filled 0.51 ; and missing, 27.79. Periodontal condition was highly deteriorated by $78.8 \%$ of teeth with loss of clinical insertion larger than $3.5 \mathrm{~mm}, 15.2 \%$ of code $4 ; 36.5 \%$ of code 3 ; and $48.5 \%$ of code 2 of PSR. The injuries of soft tissues affected $52.5 \%$ of the sample, the vascular type (venous lake) being more prevalent with $25 \%$, followed by $23.21 \%$ of denture stomatitis. 51.3\% was completely toothless. As for distribution of the tooth, in maxilla and mandible, the most frequent finding was Kennedy's Class I (50\% and 63.9\%). Only $43.6 \%$ was using their acrylic prostheses. We found an extremely damaged condition of oral health in this group, with small possibilities of being recovered, principally due to the diminished systemic conditions that they present and to the lack of coverage of the programs of oral health for these persons.

KEY WORDS: frail elderly, diagnosis, oral, bucal. 


\section{REFERENCIAS BIBLIOGRÁFICAS}

Applegate, O. C. The rationale of partial denture choice. J. Prosthetic Dent., 10:891-907, 1960.

Awano, S.; Ansai, T.; Takata, Y.; Soh, I.; Akifusa, S.; Hamasaki, T.; Yoshida, A.; Sonoki, K.; Fujisawa, K. \& Takehara, T. Oral health and mortality risk from pneumonia in the elderly. J. Dent. Res., 87(4):334-9, 2008.

Azarpazhooh, A. \& Leake, J. L. Systematic review of the association between respiratory diseases and oral health. J. Periodontol., 77(9):1465-82, 2006.

de Castellucci Barbosa, L.; Ferreira, M. R.; de Carvalho Calabrich, C. F.; Viana, A. C.; de Lemos, M. C. \& Lauria, R. A. Edentulous patients' knowledge of dental hygiene and care of prostheses. Gerodontology, 25(2):99-106, 2008.

Espinoza, I.; Rojas, R.; Aranda, W. \& Gamonal, J. Prevalence of oral mucosal lesions in elderly people in Santiago, Chile. J. Oral Pathol. Med., 32(10):571-5, 2003.

Kirkwood, T. B. \& Ritter, M. A. The interface between ageing and health in man. Age Ageing, 26(Supp/ 4):9-14, 1997.

Mariño, R.; Cueto, A.; Badenier, O.; Acevedo, R. \& Moya R. Situación y factores de riesgo en salud bucodental en adultos de 65 a 74 años en población de la región de Valparaíso, Chile. Valparaíso, Universidad de Valparaíso, 2007.

Meurman, J. H. \& Hämäläinen, P. Review article Oral health and morbidity - implications of oral infections on the elderly. Gerodontology, 23(1):3-16, 2006.

Ministerio de Salud (MINSAL). Resultados I Encuesta de Salud, Chile 2003. Disponible en: http://epi.minsal.cl/epi/ html/invest/ENS/InformeFinalENS.pdf

Nunez, M. L. \& Aspillaga, M. P. Diagnóstico de salud oral en personas mayores postradas inscritas en el Centro de Salud La Florida de la comuna de Talca. Escuela de Odontología , Talca, Chile, Universidad de Talca, 2007. Disponible en: $\boldsymbol{h t t p} / / / d s p a c e . u t a l c a . c l / h a n d l e / 1950 / 4865$.

Nunez, M. L. \& Quinteros, M. E. Diagnóstico en Salud Oral en pacientes postrados inscritos en el Consultorio Dr. José D. Astaburuaga, Talca. Escuela de Odontología , Talca, Chile, Universidad de Talca, 2006. Disponible en: http://di.utalca.cl/docs/pdf/Memoria-2005-2006.pdf

Organización Mundial de la Salud (OMS). Clasificación In- ternacional del Funcionamiento de la Discapacidad y de la Salud. Madrid, Organización Mundial de la Salud, 2001.

Petersen, P. E. \& Yamamoto, T. Improving the oral health of older people: the approach of the WHO global oral health programme. Community Dent. Oral Epidemiol., 33(2):8192, 2005.

Ribeiro, D. G.; Pavarina, A. C.; Giampaolo, E. T.; Machado, A. L.; Jorge, J. H. \& Garcia, P. P. Effect of oral higiene education and motivation on removable partial denture wearers: longitudinal study. Gerodontology, 26(2):1506, 2009.

SENAMA-INTA. Primer estudio de la dependencia en Chile, 2009. Disponible en: http://www. senama.cl/archivos/ estudiodependencia.pdf

Shimoyama, K.; Chiba, Y. \& Suzuki, Y The effect of awareness on the outcome of oral health performed by home care service providers. Gerodontology, 24(4):20410, 2007.

Superintendencia de Salud. Perfil Epidemiológico del Adulto Mayor en Chile, 2006. Disponible en: http:// www.supersalud.gob.cl/documentacion/569/w3-article4020.html

Wilson, W.; Taubert, K. A.; Gewitz, M.; Lockhart, P. B.; Baddour, L. M.; Levison, M.; Bolger, A.; Cabell, C. H.; Takahashi, M.; Baltimore, R. S.; Newburger, J. W.; Strom, B. L.; Tani, L. Y.; Gerber, M.; Bonow, R. O.; Pallasch, T.; Shulman, S. T.; Rowley, A. H.; Burns, J. C.; Ferrieri, P.; Gardner, T.; Goff, D.; Durack, D. T. \& American Heart Association. Prevention of infective endocarditis: guidelines from the American Heart Association: a guideline from the American Heart Association Rheumatic Fever, Endocarditis and Kawasaki Disease Committee, Council on Cardiovascular Disease in the Young, and the Council on Clinical Cardiology, Council on Cardiovascular Surgery and Anesthesia, and the Quality of Care and Outcomes Research Interdisciplinary Working Group. J. Am. Dent. Assoc., 139 (Suppl):3S24S, 2008.

Dirección para correspondencia:

Alfredo Cueto U.

Subida Carvallo 211

Recibido :16-04-2012

Playa Ancha

Aceptado:29-05-2012

Valparaíso

CHILE

Email: alfredocuetourbina@yahoo.es 